\title{
The crossover from propagating to strongly scattered acoustic modes of glasses observed in densified silica
}

\author{
E Courtens, M Foret, B Hehlen, B Rufflé and R Vacher \\ Laboratoire des Verres, UMR 5587 CNRS, Université Montpellier 2, F-34095 Montpellier, France
}

Received 23 January 2003, in final form 31 January 2003

Published 10 March 2003

Online at stacks.iop.org/JPhysCM/15/S1279

\begin{abstract}
Spectroscopic results on low-frequency excitations of densified silica are presented and related to characteristic thermal properties of glasses. The end of the longitudinal acoustic branch is marked by a rapid increase of the Brillouin linewidth with the scattering vector. This rapid growth saturates at a crossover frequency $\Omega_{\text {co }}$ which nearly coincides with the centre of the boson peak. The latter is clearly due to additional optic-like excitations related to nearly rigid $\mathrm{SiO}_{4}$ librations as indicated by hyper-Raman scattering. Whether the onset of strong scattering is best described as resulting from hybridization of acoustic modes with these librations, from their elastic scattering (Rayleigh scattering) on the local excitations, or from soft potentials remains to be established.
\end{abstract}

\section{Introduction}

Studying some papers in this Special Issue, or judging from recent review articles $[1,2]$, the reader will find that rather different views regarding the fate of high-frequency acoustic-like modes in glasses are still being presented. Essentially, the controversy has to do with the length scale beyond which the continuous homogeneous medium approximation breaks down in glasses. One group essentially claim that plane acoustic waves propagate with wavelengths down to the near-atomic scale in a large variety of glasses [2]. The other group, to which we belong, believe that plane waves cannot be reasonable approximations to eigenmodes for wavevectors beyond a crossover value $q_{\mathrm{co}}$ [1]. This $q_{\mathrm{co}}$ is such that $2 \pi / q_{\mathrm{co}}$ is considerably larger than the structural units of usual glasses, for example than the $\mathrm{SiO}_{4}$ tetrahedra in vitreous silica, $\mathrm{v}-\mathrm{SiO}_{2}$. As shown below, this can be demonstrated in the case of densified silica. However, on the basis of all the available information, it seems to be a reasonable hypothesis for many glasses. This is not a side issue but rather a central question for anyone with an interest in the long-range structure of these important materials. It is also crucial for the understanding of macroscopic properties that reflect the disorder. For example, it is well known that the low-temperature thermal conductivity $\kappa(T)$ of insulators is controlled by the mean free path $\ell$ of propagating acoustic waves [3,4]. As the temperature $T$ is raised from very small values, say $0.1 \mathrm{~K}$, waves of higher frequency $\Omega$ participate in the conductivity with a nearly constant $\ell$, 
so $\kappa$ increases. However, the thermal conductivity of glasses usually shows a well pronounced plateau for $T \sim 10 \mathrm{~K}$, corresponding to dominant phonons around $1 \mathrm{THz}-$ see, e.g., [4-6] or a chapter in [7]. The traditional interpretation of this feature is that the plateau corresponds to an upper limit $\Omega_{\text {co }}$ for the frequency $\Omega$ of propagating plane waves. In other words, the plateau corresponds to a crossover where $\ell \rightarrow 0$ for the dominant phonons at the plateau temperature.

As the question is of importance, it is natural to turn to spectroscopy to shed light on it. Spectroscopy has established that in strong glasses, such as $\mathrm{v}-\mathrm{SiO}_{2}$, acoustic waves propagate energy up to rather high frequencies. This has been demonstrated with pulse experiments up to $\simeq 0.3 \mathrm{THz}$ [8]. Low-temperature tunnelling experiments also revealed in $\mathrm{v}-\mathrm{SiO}_{2}$ a linear dispersion, $\Omega=v q$, and narrow linewidths $\Gamma$ up to at least $\Omega / 2 \pi \simeq 0.4 \mathrm{THz}$ [9]. Here, $v$ is the velocity and $q$ is the wavevector of the quasi-plane waves. For such waves, the spectrum has a half-width $\Gamma$ which, in $\operatorname{rad~s}^{-1}$, is $v / 2 \ell$, where $\ell$ is the energy mean free path. For quasiplane waves, $\Gamma$ is much smaller than $\Omega$. The recent development of high-resolution inelastic $\mathrm{X}$-ray spectroscopy (IXS) in principle allows one to observe the spectrum of high-frequency acoustic waves by means of X-ray Brillouin scattering [2]. Here the experimental possibilities are however limited to sufficiently high spectral frequencies $\omega$ and scattering vectors $Q$. This is presumably why the controversy arose in the first place [10-12]. Indeed, many publications claimed that sound propagates at frequencies far above the earlier expectations for $\Omega_{\mathrm{co}}[11,13-$ $19]$ - this without being really able to perform the decisive spectroscopy around $\Omega_{\text {co }}$, as it is located too low in $\omega$ to be accessible. If such claims were to be valid, one would have a very hard time explaining the observations of well defined $\kappa(T)$ plateaus in glasses $[4,5,20]$.

In our view, the above claims result from misinterpretations of the spectroscopic data. This will be explained for one particular glass below. Thus, in our opinion the real issue is not whether the crossover frequency $\Omega_{\text {co }}$ exists, which it probably does in many cases, but rather what the mechanism that produces it is. Early work assumed that Rayleigh scattering by disorder (whether disorder in masses or in force constants) would be sufficient to produce a crossover $[4,5]$. Rayleigh scattering by point defects leads to the inhomogeneous broadening of plane waves with $\Gamma=A \Omega^{4}$ [21-25]. With such a high power of $\Omega$, as soon as this broadening becomes observable, the limit $\Gamma \sim \Omega$ should be quickly reached as $\Omega$ increases further. More precisely, the upper limit for plane-wave-like propagation should rather be $\ell \simeq \lambda / 2$ which amounts to $\Gamma \simeq \Omega / 2 \pi$. This corresponds to the Ioffe-Regel crossover beyond which $\Gamma \propto \Omega$ [26] and one enters the strong-scattering regime for plane waves. The limit should essentially coincide with $\Omega_{\text {co }}$. However, the prefactor $A$ appears to be too small by about an order of magnitude to lead to the expected low value for $\Omega_{\mathrm{co}}[5,20,27]$, although [6] claims that force-constant fluctuations are sufficient in $\mathrm{v}-\mathrm{SiO}_{2}$ to account for the plateau. Thus one might wish to search for other possible mechanisms. A likely one can result from the near-universal presence of another spectral feature of glasses, the so-called 'boson peak' [20, 28-30]. This peak corresponds to additional excitations which produce a hump in $C / T^{3}$ versus $T$, where $C$ is the specific heat [4]. Like the $\kappa$-plateau, this hump is located around $10 \mathrm{~K}$. It is produced by an excess over the Debye value in the density of vibrational states (DOS), $Z(\omega)$ [31]. This excess is well observed by plotting $Z(\omega) / \omega^{2}$, a quantity that should be constant in the Debye model but which also shows a hump around $1 \mathrm{THz}$ in $\mathrm{v}-\mathrm{SiO}_{2}$ and in many other glasses. The question arose of whether these boson peak (BP) excitations just correspond to the near-horizontal end of acoustic branches in the region where $q$ reaches Brillouin-zone-like dimensions [32, 33], or whether they are rather due to other modes [34], which then must be optic-like ones. A very strong case for the latter has recently been made for various forms of silica on the basis of hyper-Raman scattering (HRS) evidence [35]. This will be briefly explained below. It has also been known for a long time that in some model systems that behave as glasses, such as the mixed crystals of $\mathrm{KCN}-\mathrm{KBr}[20,30]$, the boson peak is optic-like in nature, corresponding in 
the latter case to $\mathrm{CN}$ librations. In the case of silica, the BP forms a broad diffuse band that indicates strong inhomogeneous broadening. The acoustic modes can hybridize with these low-lying optic-like modes [36, 37]. This hybridization could then produce the crossover, in which case one should expect that $\Omega_{\mathrm{co}}$ might be close to the frequency $\Omega_{\mathrm{BP}}$ of the BP maximum.

\section{Spectroscopy of densified silica glass}

To make progress on this issue, it seems that a good way to proceed is to perform high-quality Brillouin scattering spectroscopy on well selected cases. At present, only IXS allows one to investigate the waves at both the relevant frequencies and scattering vectors. To settle this question, it is necessary to clearly observe $\Omega_{\mathrm{co}}$ and the corresponding $q_{\mathrm{co}}$, the evolution of the acoustic profile, as well as the onset of the BP scattering of X-rays. In this respect, it must be emphasized that IXS still is a difficult spectroscopy with severe limitations on resolution and intensity. Firstly, it is practically impossible with current instruments to investigate scattering vectors $Q$ below $\sim 1 \mathrm{~nm}^{-1}$. Secondly, the narrowest instrumental profile allowing for sufficient intensity [38] still has an energy width around $1.5 \mathrm{meV}$ (or $\simeq 0.4 \mathrm{THz}$ ) and extended Lorentzianlike wings. Owing to the relatively strong elastic scattering of glasses, this tends to mask the weaker Brillouin signal. Finally, the signal-to-noise ratio also being small, the subtle changes in Brillouin lineshapes that indicate strong scattering might easily go unnoticed [12]. For the above reasons, the necessary spectroscopy cannot yet be performed on normal vitreous silica, $\mathrm{v}-\mathrm{SiO}_{2}$. In that case, the position of the $\kappa$-plateau around $10 \mathrm{~K}$ suggests that $\Omega_{\mathrm{co}} \simeq 4 \mathrm{meV}$ (or $\Omega_{\mathrm{co}} / 2 \pi \simeq 1 \mathrm{THz}$ ), so one expects $q_{\mathrm{co}} \simeq 1 \mathrm{~nm}^{-1}$ for the longitudinal acoustic waves of velocity $v \simeq 5900 \mathrm{~m} \mathrm{~s}^{-1}$. These values of $\Omega_{\text {co }}$ and $q_{\text {co }}$ are indeed too low for clearly observing crossover phenomena in $\mathrm{v}-\mathrm{SiO}_{2}$. To alleviate several of these difficulties, we investigated another form of silica glass, permanently densified silica d-SiO ${ }_{2}$, in which case we found that $\Omega_{\mathrm{co}} / 2 \pi \simeq 2 \mathrm{THz}$ and $q_{\mathrm{co}} \simeq 2 \mathrm{~nm}^{-1}[39,40]$.

That silica can be permanently densified when subjected to high pressures has been known for a long time [41]. The sample of $\mathrm{d}-\mathrm{SiO}_{2}$ used in all our IXS measurements was densified at $\sim 1000 \mathrm{~K}$ and $7.4 \mathrm{GPa}$ [42]. It was kindly provided by Dr M Arai. It is a clear, transparent piece, whose good homogeneity has been checked by optical Brillouin spectroscopy [43]. Its density is $\rho \approx 2.60 \mathrm{~g} \mathrm{~cm}^{-3}$, much above that of $\mathrm{v}_{-}-\mathrm{SiO}_{2}, \rho=2.20 \mathrm{~g} \mathrm{~cm}^{-3}$, and near that of crystal quartz. Intuitively, one expects that the range of inhomogeneities should be strongly reduced by densification, and hence that $q_{\mathrm{co}}$ and $\Omega_{\mathrm{co}}$ should be larger in d- $\mathrm{SiO}_{2}$ than in $\mathrm{v}-\mathrm{SiO}_{2}$. An increase of $\Omega_{\mathrm{co}}$ is indeed indicated by the $\kappa(T)$ data. The plateau seems to be around $20 \mathrm{~K}$ for $\mathrm{d}-\mathrm{SiO}_{2}$ [44], suggesting that $\Omega_{\mathrm{co}}$ will be roughly twice that of $\mathrm{v}-\mathrm{SiO}_{2}$. Also, the disturbing signal from the elastic structure factor, $S(Q, 0)$, should be reduced considerably compared to that of $\mathrm{v}-\mathrm{SiO}_{2}$ [42]. For these reasons we anticipated that the crucial region below and near $\Omega_{\text {co }}$ might be accessible to spectroscopy in $\mathrm{d}-\mathrm{SiO}_{2}$ using the current IXS capabilities.

\section{The crossover to strong scattering}

Figure 1 shows a series of IXS spectra of $\mathrm{d}-\mathrm{SiO}_{2}$ taken at small values of $Q$, up to the expected $q_{\text {co }}$ [45]. These spectra were obtained on the spectrometer ID16 at the European Synchrotron Radiation Facility in Grenoble, France. The experimental conditions are described in $[39,45]$. As shown in figure 1(a), the spectra are dominated by an elastic central peak. To increase the relative strength of the inelastic contribution, the sample is placed at an elevated temperature which increases the thermal population of the acoustic modes. However, $\mathrm{d}-\mathrm{SiO}_{2}$ relaxes towards $\mathrm{v}-\mathrm{SiO}_{2}$ if $T$ is too high. We found that a good compromise is $T=565 \mathrm{~K}$, as at that 


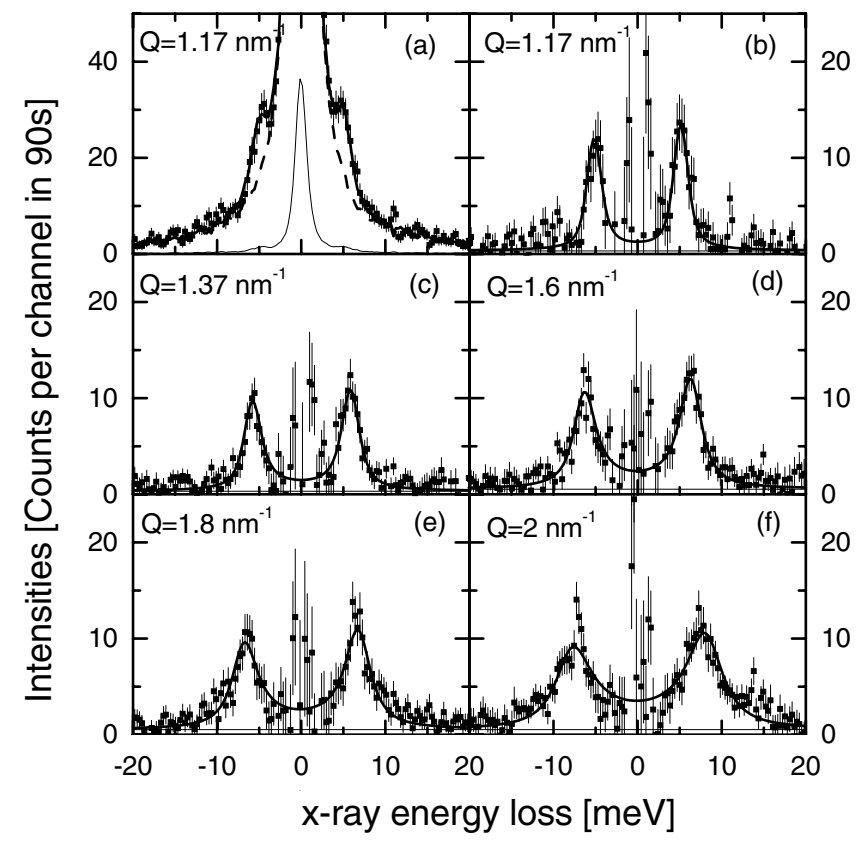

Figure 1. IXS spectra of d-SiO . (a) A full spectrum with its central part, divided by 20, shown by the solid line. The dashed curve shows the adjusted elastic contribution. (b)-(f) The inelastic part for five different scattering vectors with the solid curves showing the DHO fits. The fitted elastic component was subtracted from the data (see the text). The level of the electronic dark counts is shown by the baselines.

value the density does not change over periods of weeks [43]. To obtain significant information on the inelastic Brillouin doublet, it is necessary to have an instrumental lineshape which is extremely clean far into its wings. This has been the case in this particular experiment [45]. The instrumental lineshape was determined for each analyser using the signal of polymethyl methacrylate at $20 \mathrm{~K}$. Very clean Voigt-like profiles were obtained with half-widths at halfmaximum of $\simeq 0.75 \mathrm{meV}$. The spectra can thus be fitted to the sum of an elastic line of integrated intensity $I_{\mathrm{CP}}$ plus a suitable spectral function $S(Q, \omega)$. Below the crossover and in the absence of any other information, it is reasonable to select for $S(Q, \omega)$ a standard damped harmonic oscillator (DHO) response function. In this way one obtains a frequency $\Omega$, a halfwidth $\Gamma$, and an integrated intensity $I_{\mathrm{DHO}}$ independently of any other assumption. Of course the linewidth will be a combination of a homogeneous (or lifetime) broadening $\Gamma_{\text {hom }}$ and of any inhomogeneous broadening $\Gamma_{\text {inh }}$ that might result from the approach to the crossover. To evaluate the spectra, the response function must be convoluted with the instrumental lineshape. To extract significant inelastic widths it is necessary to take into account the collection angles that are fixed by the slits placed in front of the analysers. These give a spread in the scattering vectors, $\Delta Q= \pm 0.18 \mathrm{~nm}^{-1}$. The fitting procedure sums over surface elements of the slit, each at its own $Q$, using $\mathrm{d} \Omega / \mathrm{d} Q=v_{\mathrm{g}}$, where $v_{\mathrm{g}}$ is the group velocity which is determined iteratively. Figures 1(b)-(f) represent the pure inelastic part that remains after subtraction of the central peak of adjusted strength $I_{\mathrm{CP}}$. The solid curves are the convoluted DHO fits. The half-width $\Gamma$ obtained for $Q=2 \mathrm{~nm}^{-1}$ is somewhat larger than the corresponding $\Omega / 2 \pi$. Hence, that value of $Q$ is already in the crossover region [39]. This confirms the estimate made on the basis of the $\kappa$-plateau [44] and it shows that in $\mathrm{d}-\mathrm{SiO}_{2}$ the crossover region is accessible. 


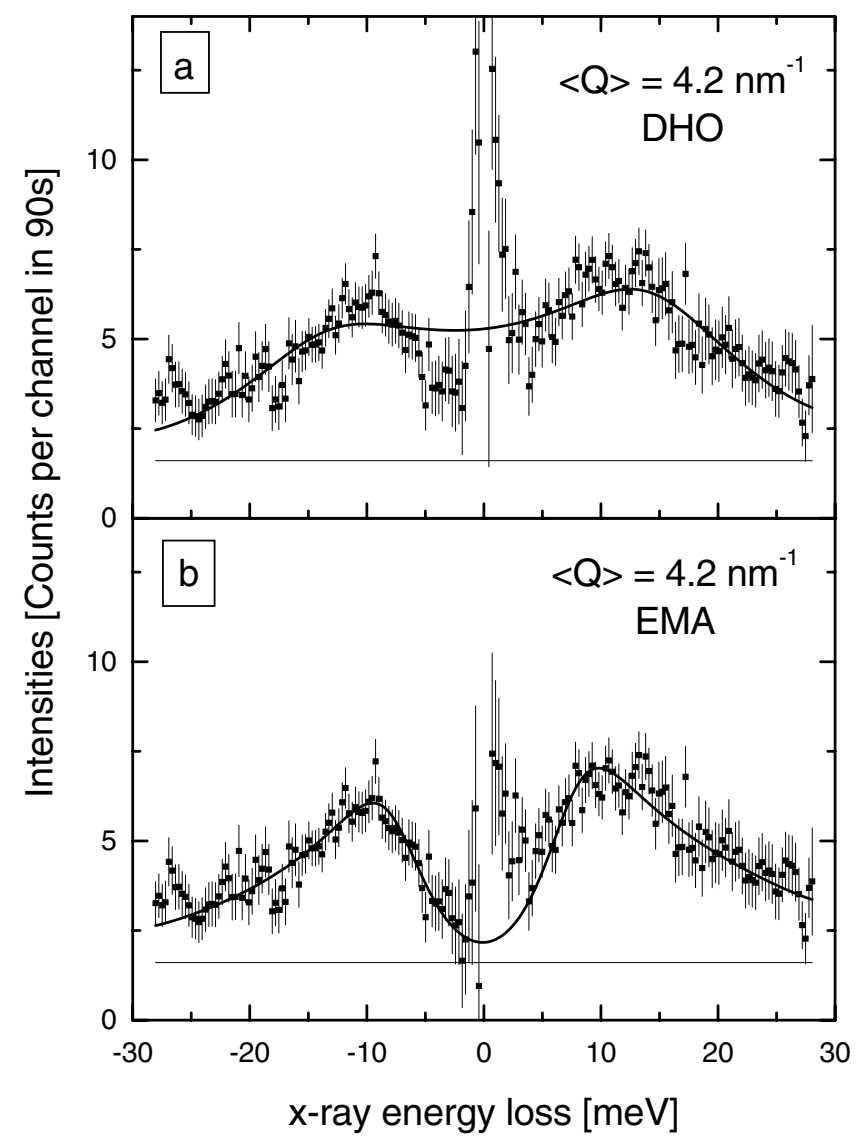

Figure 2. The inelastic part of the IXS spectra of $\mathrm{d}-\mathrm{SiO}_{2}$ for $Q>q_{\mathrm{co}}$. The solid curves are fits to the DHO (a) and EMA (b) spectral profiles. The dark-count baselines are shown.

Figure 2 shows a spectrum obtained for $Q>q_{\mathrm{co}}$. Beyond the crossover the spectra evolve quite slowly with $Q$. What is shown in figure 2 is the sum of spectra obtained at $Q=4,4.2$, and $4.4 \mathrm{~nm}^{-1}$ in the course of accumulating the spectra shown in figure 1 . In this way, the total accumulation time in figure 2 is nearly one week. This summation improves the signal-to-noise ratio without appreciably changing the spectral shape. For $Q>q_{\mathrm{co}}$, one does not expect that the DHO can be a reasonable approximation and, indeed, the DHO fit in figure 2(a) is not satisfactory. In particular, it gives a contribution near $\omega=0$ which is much higher than what the data suggest, this in spite of the freely adjustable $I_{\mathrm{CP}}$ which tries to compensate for it. The reason is that for $q>q_{\text {co }}$ there are no eigenmodes with a well defined $q$-value. For a given $\Omega>\Omega_{\text {co }}$, the eigenmodes consist in a broad superposition of plane waves in $q$-space. The measurement being performed at a fixed scattering vector $Q$, the spectrum is the projection on $Q$ of the appropriate Fourier components for all $\Omega$ s [46]. On the other hand, at low frequencies $\omega$, the modes with $\Omega=\omega$ are well defined plane waves of a given $q \ll q_{\mathrm{co}}$. These do not have Fourier components at $Q \gg q_{\mathrm{co}}$ and for this reason the spectrum dips at its centre. To approximately describe this situation, one can make an EMA (effectivemedium approximation) in which the inhomogeneity is described by a frequency-dependent velocity, $v(\omega)$, and linewidth, $\Gamma(\omega)$. Figure 2(b) shows the result of such an EMA adjustment as described in [39]. One should note that once the parameters of the EMA are fixed, they allow prediction of both the spectral shape and the strength at all $Q$-values. 


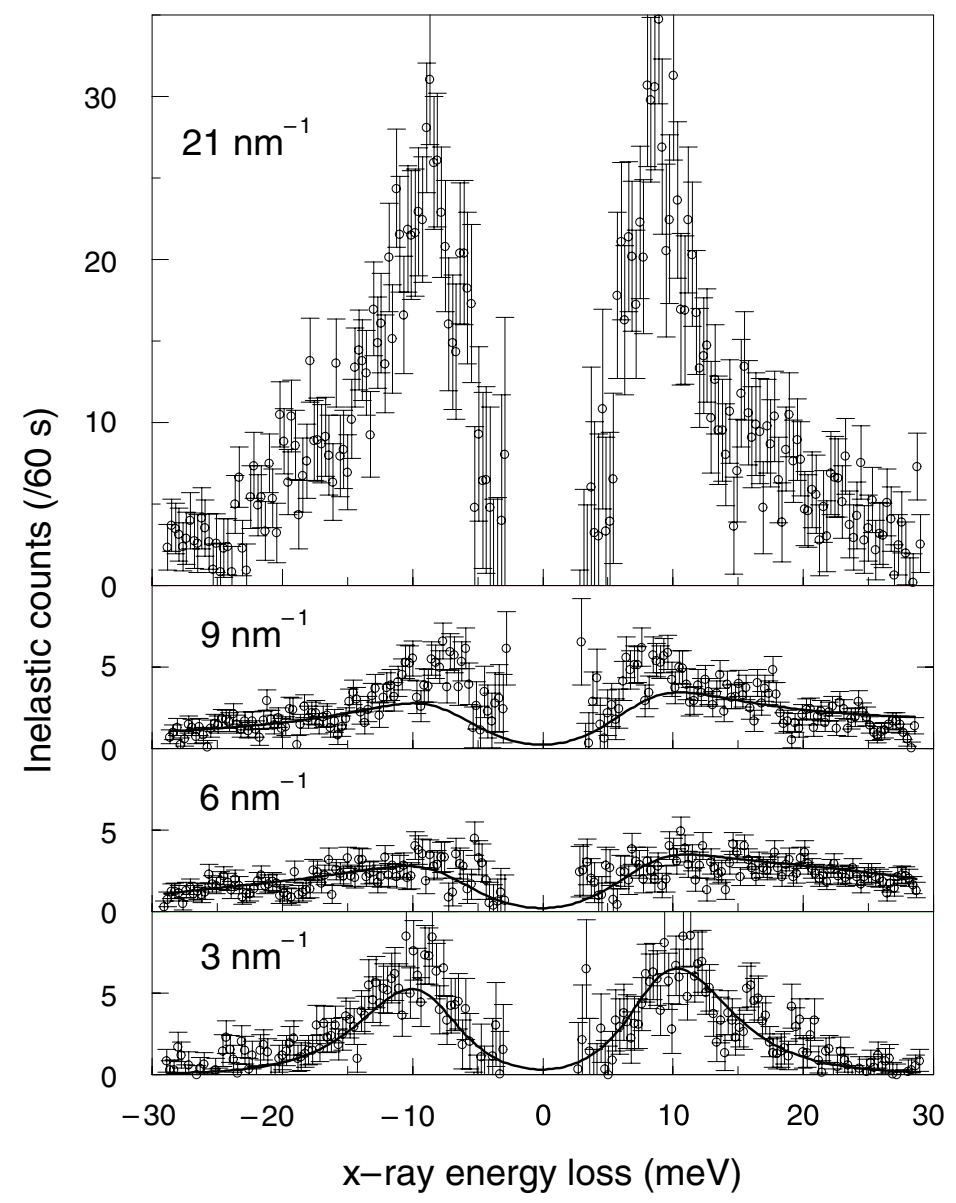

Figure 3. The inelastic part of the IXS spectra at scattering vectors above the crossover. The solid curves show the prediction of the EMA models with the parameters obtained from the fits in [39]. The dark counts have been subtracted.

Figure 3 shows the evolution of the IXS spectra deeper into the strong-scattering regime, i.e. for $Q$ growing beyond $q_{\text {co }}$ [40]. The spectra are shown on a single relative scale, uncorrected for the atomic form factors. The form factors reduce the observed intensity by $14 \%$ at $9 \mathrm{~nm}^{-1}$ and by nearly a factor of two at $21 \mathrm{~nm}^{-1}$. The solid curves are the predictions of the same EMA as in figure 2, taking into account the effect of the atomic form factors. At $9 \mathrm{~nm}^{-1}$, an extra signal appears on top of the EMA which then grows rapidly to become overwhelming at $21 \mathrm{~nm}^{-1}$. This is the scattering from the BP excitations. These BP spectra are similar to those that have been observed with inelastic neutron scattering [31]. They have, in that case, been discussed within an 'incoherent approximation' [47]. This essentially amounts to assuming that $2 \pi / Q$ is small compared to the coherence length of the vibrating modes so that on the average one observes single vibrating units that can be thought of as moving incoherently with respect to one another. In that case, the quantity which is observed is essentially $\propto n(\omega) Z(\omega) / \omega$, where $n(\omega)$ is the Bose factor. This is easily converted to $Z(\omega) / \omega^{2}$, as displayed e.g. in [48]. The rapid increase of intensity in figure 3 is consistent with an approximate growth with $Q^{2}$ [31]. The main point is that the BP maximum, $\Omega_{\mathrm{BP}}$, is seen to practically coincide with $\Omega_{\mathrm{co}}$. It is thus of considerable interest to investigate the origin of these BP modes. 


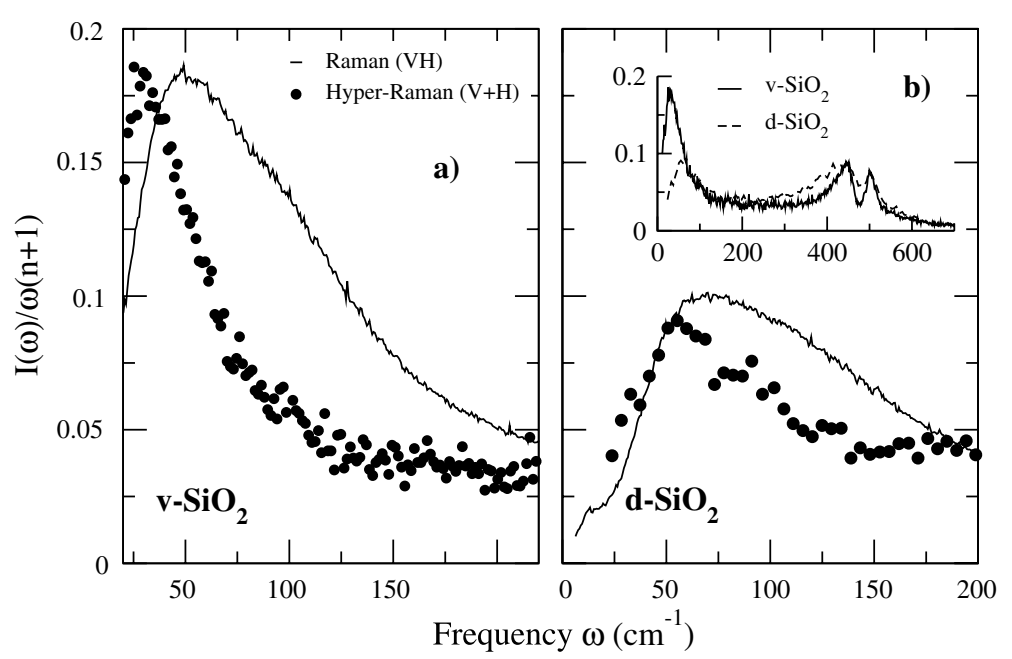

Figure 4. Raman and HRS spectra of $\mathrm{v}-\mathrm{SiO}_{2}$ (a) and d-SiO 2 (b) in the boson peak region. The inset in (b) shows HRS spectra of $\mathrm{v}-\mathrm{SiO}_{2}$ and $\mathrm{d}-\mathrm{SiO}_{2}$ over a frequency range which allows the observation of the first TO and LO modes. The data are drawn on comparable relative scales.

\section{The origin of the boson peak for silica glasses}

The nature of the BP in glasses has long been discussed. Qualitatively similar BP spectra are often observed in various spectroscopies at scattering vectors $Q$ which differ by orders of magnitude. This suggests that these excitations are quasi-local. However, a difficulty arose for silica from the fact that the inelastic neutron scattering (INS) spectra and the Raman scattering (RS) ones are quantitatively quite different [33]. If the same modes are observed in both spectroscopies, one would expect that the RS intensity is $\propto Z(\omega) n(\omega) C(\omega) / \omega$, where $Z(\omega)$ is the DOS observed with neutrons [49]. The coupling coefficient $C(\omega)$ should be $\propto \omega^{2}$ for active acoustic modes [50] and should be constant for active optic ones [49]. Instead of that, the experimental determination gives approximately $C(\omega) \propto \omega^{1}$ in $\mathrm{v}^{-\mathrm{SiO}_{2}}$ [33]. To clarify this situation, we performed HRS spectroscopy on various forms of silica glasses, in particular $\mathrm{v}-\mathrm{SiO}_{2}$ and $\mathrm{d}-\mathrm{SiO}_{2}$ [35]. HRS spectroscopy is a non-linear scattering spectroscopy in which two incoming photons give one scattered photon [51]. It obeys selection rules which are different from those governing RS and infrared (IR) absorption. In particular, if the effective symmetry is that of isotropic media, $\infty \infty \mathrm{m}$, then there is mutual exclusion between RS and HRS activities. This means that acoustic modes, which are active in Brillouin scattering, are not active in HRS. On the other hand, one can consider the vibrational modes of local units of lower symmetry, for example the $\mathrm{SiO}_{4}$ tetrahedra. The rigid rotations of undistorted tetrahedra, which have a spherically symmetric polarizability, are not active in RS but they happen to be active in HRS [52].

Some of the HRS results are illustrated in figure 4. One sees immediately that the RS and HRS spectra are very different. In addition, although the HRS signal is very small, the HRS activity is much larger than the RS one. The quantitative comparison is explained in [35] where it is shown that the BP modes are mostly RS inactive while they are HRS active. This is a first indication that the BP modes are optic-like. One also notes the quasi-absence of a BP in IR [35], another selection rule which shows that the BP is essentially non-polar. Comparing with $Z(\omega) / \omega^{2}$ obtained in INS, one finds a nearly perfect overlap with the HRS spectra [35]. This obviously indicates that for HRS the coupling constant $C(\omega)$ is $\propto \omega^{0}$, which is a second 
proof that the relevant modes are optic-like. On the basis of INS it was already noted that rigid $\mathrm{SiO}_{4}$ tetrahedra librations are likely to lie at the origin of the BP excitations [31]. The authors of [31] remarked that the $Q$-dependence of the scattered spectra could be qualitatively reproduced by a model taking into account the coupled rotations of five rigid $\mathrm{SiO}_{4}$ tetrahedra. In this respect it is interesting to remark that of all the modes of a tetrahedral 'molecule', the only ones which have the required selection rules, namely to be RS and IR inactive and HRS active, are the rigid rotations [52].

Interestingly, nearly rigid tetrahedra rotations lie at the origin of the $\beta \rightarrow \alpha$ transition in crystal quartz which occurs at around $570{ }^{\circ} \mathrm{C}$. The soft mode of this transition is silent in RS but active in HRS [53]. Moreover, the extrapolation of that HRS measurement shows that at $T \simeq 1100^{\circ} \mathrm{C}$, the frequency of the soft mode is approximately located at the position of the $\mathrm{BP}$ of $\mathrm{v}-\mathrm{SiO}_{2}$. That value of $T$ corresponds to the $T_{\mathrm{g}}$ of $\mathrm{v}-\mathrm{SiO}_{2}$. Therefore the $\mathrm{BP}$ in the glass can be viewed as a frozen soft mode. As the freezing is taking place far from the actual transition, the correlation length of the mode is expected to be relatively short. In $\mathrm{d}-\mathrm{SiO}_{2}$, the densification from 2.2 to $2.60 \mathrm{~g} \mathrm{~cm}^{-3}$ obviously should arrest the collective rotations which have the largest spatial extent. The low-frequency part of the $\mathrm{BP}$ of $\mathrm{v}-\mathrm{SiO}_{2}$ just disappears in going to $\mathrm{d}-\mathrm{SiO}_{2}$. This is clearly seen in figure 4(b), in particular in the inset which shows spectra of $\mathrm{v}-\mathrm{SiO}_{2}$ and $\mathrm{d}-\mathrm{SiO}_{2}$ on the same relative scale. This suggests that the collective excitations with the largest spatial extent also have the lowest frequencies, just as for a soft mode near a structural transition. This inset of figure 4 also shows the lowest TO-LO modes. The TO develops a broad low- $\omega$ wing in $\mathrm{d}-\mathrm{SiO}_{2}$ and this wing apparently contributes to the HRS signal in figure 4(b) at frequencies above $\sim 150 \mathrm{~cm}^{-1}$.

In view of all the above, it is now clear that the $\mathrm{BP}$ of silica is an optic-like mode related to the nearly rigid rotation of the $\mathrm{SiO}_{4}$ units. This is also confirmed by several independent recent simulations [54-56]. This BP is active in HRS, while what is observed in RS is most probably a leakage of this forbidden mode: either the tetrahedra are slightly distorted and therefore their polarizability is not fully spherically symmetric, or the scattering results from the not fully symmetric near environment of the tetrahedra. We believe that the unusual exponent found in RS for the coupling coefficient, $C(\omega) \propto \omega^{1}$, precisely reflects the average strength of this leakage of forbidden modes. As the BP does not appreciably change its spectral shape with $T$, it is obviously very strongly inhomogeneously broadened, meaning that single BP components can be very long-lived quasi-local vibrations that are distributed randomly in the volume of the glass. On the other hand, it is intuitively obvious that the $\mathrm{SiO}_{4}$ rotations will have a bilinear coupling with elastic deformations, and therefore with acoustic-like modes ${ }^{1}$. As acoustic modes become resonant with BP modes, this bilinear coupling produces hybridization which amounts to their strong scattering owing to the random spatial distribution of the BP modes. Evidence for this can be searched for in the onset of strong scattering as $\Omega$ increases towards $\Omega_{\mathrm{BP}}$.

\section{The onset of strong scattering in densified silica}

The linewidths $\Gamma$ extracted from the DHO fits shown in figure 1 are plotted in figure 5. One notices a very rapid increase of $\Gamma$ with $\Omega$. We use Matthiessen's rule that $\Gamma=\Gamma_{\text {hom }}+\Gamma_{\text {inh }}$. To evaluate $\Gamma_{\text {inh }}$ we need an estimate for $\Gamma_{\text {hom. }}$. Usually the linewidth observed in optical Brillouin scattering is purely homogeneous, barring macroscopic inhomogeneities in the density which were carefully avoided [43]. We found that in near backscattering, for $\Omega=41.5 \mathrm{GHz}$,

1 In $\beta$-quartz, there is a bilinear coupling between the gradient of the soft mode and the acoustic modes [57]. That coupling produces the incommensurate phase that appears between the $\beta$ - and $\alpha$-ones [58]. In the glass, the gradient becomes superfluous owing to the randomness. 


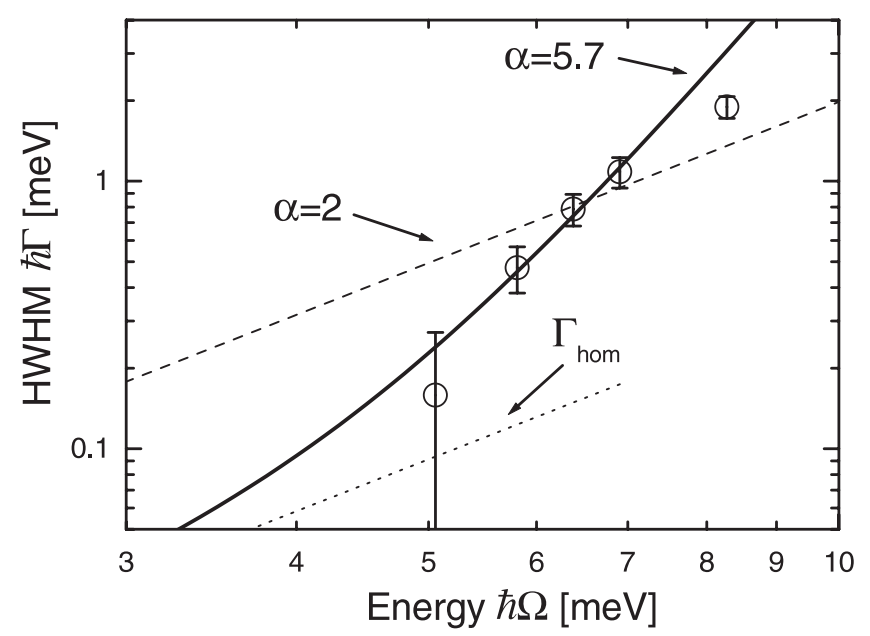

Figure 5. The half-widths at half-maximum of the IXS spectra obtained from the DHO fits shown in figure 1. They are presented as functions of $\Omega$. The estimated homogeneous broadening is shown by the dotted line labelled $\Gamma_{\text {hom }}$. The other lines are fits as described in the text.

the $\Gamma$ of $\mathrm{d}-\mathrm{SiO}_{2}$ is similar to that of $\alpha$-quartz, and this over a broad $T$-range, from 100 to $300 \mathrm{~K}$ [43]. As the homogeneous attenuation in $\alpha$-quartz is of the Akhieser type [59, 60], it is reasonable to assume that also for $\mathrm{d}-\mathrm{SiO}_{2}$ one would have approximately $\Gamma_{\text {hom }} \propto \Omega^{2}$. This gives the line marked $\Gamma_{\text {hom }}$ plotted in figure 5. It is extrapolated from the optical Brillouin value $\Gamma_{\text {hom }}=26 \pm 5 \mathrm{MHz}$ at $41.5 \mathrm{GHz}$. If there were to be appreciable relaxation contributions, as described for $\mathrm{v}-\mathrm{SiO}_{2}$ in [61], our extrapolation would certainly be an upper bound.

The measured widths are clearly above the line $\Gamma_{\text {hom }}$ and they increase with $\Omega$ faster than $\Omega^{2}$. This can be shown ad absurdum by drawing the best fit in $\Gamma \propto \Omega^{2}$. One obtains the dashed straight line marked $\alpha=2$ in figure 5. The fit gives a mean square deviation $\chi^{2}=5.4$, which is very poor indeed. On the other hand, one might attempt a power law fit $\Gamma_{\text {inh }}=A \Omega^{\alpha}$. In that case one should remark that the point measured at $\Omega>8 \mathrm{meV}$ is already in the crossover region, as discussed above. Therefore one does not expect $\Gamma_{\text {inh }}$ to continue to increase strongly for that last point, as the Ioffe-Regel saturation must be felt at that frequency. Fitting the first four points we find $\alpha=5.7 \pm 1.0$ with $\chi^{2}=0.28$. The best fit, shown by the full curve, goes clearly above the last point by an amount which is consistent with an $\Omega_{\mathrm{co}}$ that would be located around $8.5 \mathrm{meV}$.

The above evidence shows undisputably that there is a $\Gamma_{\text {inh }}$ that grows rapidly between $\Omega \sim 4$ and $\Omega \sim 8 \mathrm{meV}$ and that it tends to saturate above $8 \mathrm{meV}$. It is of interest to also draw the same information as a function of $Q$. This is shown in figure 6. Here we have plotted $\Gamma_{\text {hom }} \propto Q^{2}$. The fit of the first four points to $\Gamma_{\text {inh }}=B \Omega^{\beta}$ gives now $\beta=4.0 \pm 1.2$ with $\chi^{2}=0.62$. This difference from the power $\alpha$ above results from the dispersion, $\Omega(q)$. One must therefore be quite careful in discussing quantitatively the approach to the crossover. In fact, a more correct expression for disorder-induced broadening by Rayleigh scattering in a dispersive isotropic medium, instead of $\Gamma_{\text {inh }} \propto \Omega^{4}$, is rather [62]

$$
\Gamma_{\mathrm{inh}}=\Delta \Omega(a q)^{2}\left(\frac{\Omega a}{v_{\mathrm{g}}}\right) .
$$

Here, $\Delta$ is a dimensionless coefficient that characterizes the disorder and $a$ is a microscopic dimension. Equation (1) can also be adjusted to the first four data points in figure 6, using the phase velocity $v_{\phi}=\Omega / q$ and the group velocity $v_{\mathrm{g}}$ known from our measurement. This 


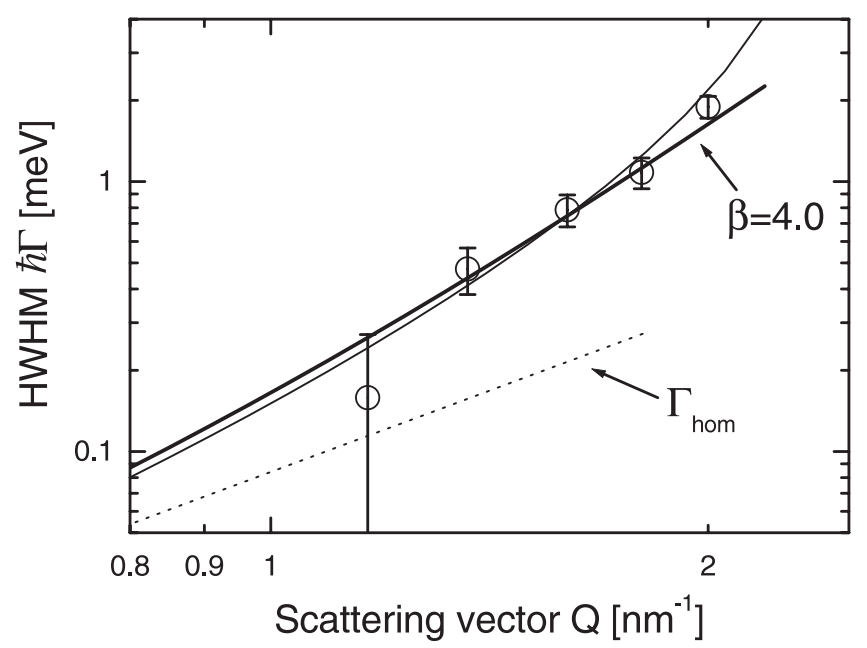

Figure 6. The same half-widths, presented as functions of $Q$. An estimated homogeneous broadening is shown by the dotted line labelled $\Gamma_{\text {hom }}$. The heavy solid line is an adjustment of the first four points to $B Q^{\beta}$. The lighter solid line is an adjustment of the same points to equation (1).

gives the lighter solid curve. With $a=1 / q_{\text {co }}=0.5 \mathrm{~nm}$, we find $\Delta=0.117 \pm 0.012 . \chi^{2}$ equals 0.66 , which is very satisfactory for this one-parameter adjustment. Knowing $\Delta$, and using $\ell^{-1}=D \Omega^{4}\left(\hbar / k_{\mathrm{B}}\right)^{4}$ as in [6], one can extract

$$
D=2 \Delta a^{3} k_{\mathrm{B}}^{4} / v_{\phi}^{2} v_{\mathrm{g}}^{2} \hbar^{4} .
$$

This gives $D=4 \mathrm{~m}^{-1} \mathrm{~K}^{-4}$. This value should be compared to $D=100 \mathrm{~m}^{-1} \mathrm{~K}^{-4}$ obtained in [6] for $\mathrm{v}-\mathrm{SiO}_{2}$. The lower value of $D$ for $\mathrm{d}-\mathrm{SiO}_{2}$ is of course consistent with the higher value of $\Omega_{\text {co }}$. Following the analysis of [6], this value is sufficient to account for the $\kappa(T)$ plateau of $\mathrm{d}-\mathrm{SiO}_{2}$. However, whether the size of $D$ can be explained as in [6] requires additional information about $\mathrm{d}-\mathrm{SiO}_{2}$ which does not seem available so far.

\section{Conclusions}

The main conclusion of this study is that a crossover to strong scattering is definitely observed in $\mathrm{d}-\mathrm{SiO}_{2}$. It is marked by a very rapid growth of the Brillouin linewidth as the Brillouin frequency approaches $\Omega_{\text {co }}$. This result disagrees with previous claims that sound propagates up to very high frequencies in silica $[11,14,19]$, but it is in line with more recent observations on other strong glasses $[63,64]$. For $\mathrm{v}-\mathrm{SiO}_{2}$, it also agrees with the result of a high-quality simulation [65]. More generally, our experience shows that IXS alone cannot prove or disprove the existence of an $\Omega_{\mathrm{co}}$ if the latter is located too low in frequency. In this respect, other IXSbased claims $[13,15-18]$ that sound propagates up to very high frequencies in glasses that are otherwise known to show a well defined $\kappa(T)$ plateau should be treated with great caution. Only when the plateau is very weak and the BP almost absent, like in calcium potassium nitrate CKN [64], may it be reasonable to think that acoustic waves propagate above the BP.

A second strong conclusion is that the position of $\Omega_{\text {co }}$ nearly coincides with the centre of the boson peak, $\Omega_{\mathrm{BP}}$. Our observations are in line with a boson peak that consists of additional modes corresponding to nearly rigid tetrahedra rotations. These modes are strongly inhomogeneously broadened and their eigenvectors should be highly disordered. 
A third conclusion, not as strong as the previous ones because only longitudinal excitations have been measured, is that the size of the increase of $\Gamma_{\text {inh }}$ with $\Omega$ or $q$ is in principle sufficient to account for the thermal conductivity plateau.

The remaining question is that of the exact origin of this crossover. Is it due to Rayleigh scattering, as advocated by many authors including ourselves in earlier times [10, 12, 39], is it rather caused by hybridization with the boson peak, or should it be described by the softpotential model [36, 66-68]? In this respect one must remark that the random forces invoked in [6] to explain the crossover in terms of Rayleigh scattering are precisely the $\mathrm{Si}-\mathrm{O}-\mathrm{Si}$ bending forces which are the dominant restoring forces for tetrahedra rigid rotations. Presumably, the soft-potential and two-level systems are also related to nearly rigid tetrahedra motions. From the discussion in the previous section it seems that the shape of the $Q$-dependence of $\Gamma_{\text {inh }}$ will not be able to settle this central issue. However, the important forces have been quite well identified. A critical theoretical analysis of the situation will be necessary to make further progress.

\section{Acknowledgments}

The authors thank Dr M Arai for the excellent sample of $\mathrm{d}-\mathrm{SiO}_{2}$ without which these investigations would not have been possible. They also thank the staff of ESRF, and in particular Dr G Monaco and Dr C Masciovecchio for their expert supervision of the various IXS experiments. Professor K Inoue and Dr Y Yamanaka are thanked for their invaluable collaboration in the hyper-Raman measurements performed in Sapporo. Professor A K Tagantsev is thanked for the derivation of the Rayleigh scattering expression for a dispersive isotropic medium.

\section{References}

[1] Courtens E, Foret M, Hehlen B and Vacher R 2001 Solid State Commun. 117187

[2] Ruocco G and Sette F 2001 J. Phys.: Condens. Matter 139141

[3] Kittel C 1949 Phys. Rev. 75972

[4] Zeller R C and Pohl R O 1971 Phys. Rev. B 42029

[5] Graebner J E, Golding B and Allen L C 1986 Phys. Rev. B 345696

[6] Raychaudhuri A K 1989 Phys. Rev. B 391927

[7] Phillips W A (ed) 1981 Amorphous Solids: Low Temperature Properties (Berlin: Springer)

[8] Zhu T C, Maris H J and Tauc J 1991 Phys. Rev. B 444281

[9] Rothenfusser M, Dietsche W and Kinder H 1984 Phonon Scattering in Condensed Matter ed W Eisenmenger, K Lassmann and S Döttinger (Berlin: Springer) p 419

[10] Foret M, Courtens E, Vacher R and Suck J-B 1996 Phys. Rev. Lett. 773831

[11] Benassi P, Krisch M, Masciovecchio C, Mazzacurati V, Monaco G, Ruocco G, Sette F and Verbeni R 1996 Phys. Rev. Lett. 773835

[12] Foret M, Courtens E, Vacher R and Suck J-B 1997 Phys. Rev. Lett. 784669

[13] Masciovecchio C, Ruocco G, Sette F, Krisch M, Verbeni R, Bergmann U and Soltwisch M 1996 Phys. Rev. Lett. 763356

[14] Masciovecchio C, Ruocco G, Sette F, Benassi P, Cunsolo A, Krisch M, Mazzacurati V, Mermet A, Monaco G and Verbeni R 1997 Phys. Rev. B 558049

[15] Masciovecchio C, Monaco G, Ruocco G, Sette F, Cunsolo A, Krisch M, Mermet A, Soltwisch M and Verbeni R 1998 Phys. Rev. Lett. 80544

[16] Fioretto D, Buchenau U, Comez L, Sokolov A, Masciovecchio C, Mermet A, Ruocco G, Sette F, Willner L, Frick B, Richter D and Verdeni L 1999 Phys. Rev. E 594470

[17] Ruocco G, Sette F, Di Leonardo R, Fioretto D, Krisch M, Lorenzen M, Masciovecchio C, Monaco G, Pignon F and Scopigno T 1999 Phys. Rev. Lett. 835583

[18] Masciovecchio C, Mermet A, Ruocco G and Sette F 2000 Phys. Rev. Lett. 851266 
[19] Pilla O, Cunsolo A, Fontana A, Masciovecchio C, Monaco G, Montagna M, Ruocco G, Scopigno T and Sette F 2000 Phys. Rev. Lett. 852136

[20] Randeria M and Sethna J P 1988 Phys. Rev. B 3812607

[21] Carruthers P 1961 Rev. Mod. Phys. 3392

[22] Klemens P G 1958 Solid State Physics vol 7, ed F Seitz and D Turnbull (New York: Academic) p 1

[23] Klemens P G 1955 Proc. R. Soc. A 681113

[24] Pomeranchuk I 1942 J. Phys. (USSR) 6237

[25] Maradudin A A 1966 Solid State Physics vol 18, ed F Seitz and D Turnbull (New York: Academic) p 273

[26] Ioffe A F and Regel A R 1960 Prog. Semicond. 4237

[27] Jones D P, Thomas N and Phillips W A 1977 Phil. Mag. B 383723

[28] Dreyfus B, Fernandez N C and Maynard R 1968 Phys. Lett. A 26647

[29] Yu C C and Freeman J J 1987 Phys. Rev. B 367620

[30] Grannan E R, Randeria M and Sethna J P 1990 Phys. Rev. B 417799

[31] Buchenau U, Prager M, Nücker N, Dianoux A J, Ahmad N and Phillips W A 1986 Phys. Rev. B 345665

[32] Masciovecchio C, Mazzacurati V, Monaco G, Ruocco G, Scopigno T, Sette F, Benassi P, Cunsolo A, Fontana A, Krisch M, Mermet A, Montagna M, Rossi F, Sampoli M, Signorelli G and Verbeni R 1999 Phil. Mag. B 79 2013

[33] Fontana A, Dell'Anna R, Montagna M, Rossi F, Viliani G, Ruocco G, Sampoli M, Buchenau U and Wischnewski A 1999 Europhys. Lett. 4756

[34] Buchenau U, Nücker N and Dianoux A J 1984 Phys. Rev. Lett. 532316

[35] Hehlen B, Courtens E, Vacher R, Yamanaka A, Kataoka M and Inoue K 2000 Phys. Rev. Lett. 845355

[36] Karpov V G and Parshin D A 1985 Sov. Phys.-JETP 611308

[37] Klinger M and Kosevich A M 2001 Phys. Lett. A 280365

[38] Verbeni R, Sette F, Krisch M, Bergmann U, Gorges B, Halcoussis C, Martel K, Masciovecchio C, Ribois J F, Ruocco G and Sinn H 1996 J. Synchrotron Radiat. 362

[39] Rat E, Foret M, Courtens E, Vacher R and Arai M 1999 Phys. Rev. Lett. 831355

[40] Foret M, Vacher R, Courtens E and Monaco G 2002 Phys. Rev. B 66024204

[41] Bridgman P W and Šimon I 1953 J. Appl. Phys. 24405

[42] Inamura Y, Arai M, Kitamura N, Bennington S M and Hannon A C 1998 Physica B 241-243 903

[43] Rat E 1999 PhD Thesis University of Montpellier, France

[44] Zhu Da-Ming 1994 Phys. Rev. B 506053

[45] Rufflé B, Foret M, Courtens E, Vacher R and Monaco G 2003 Phys. Rev. Lett. at press

[46] Vacher R, Courtens E and Foret M 1999 Phil. Mag. 791763

[47] Buchenau U 1985 Z. Phys. B 58181

[48] Inamura Y, Arai M, Yamamuro O, Inaba A, Kitamura N, Omoto T, Matsuo T, Bennington S M and Hannon A C 1999 Physica B 263/264 299

[49] Shuker R and Gammon R W 1970 Phys. Rev. Lett. 25222

[50] Martin A J and Brenig W 1974 Phys. Status Solidi b 64163

[51] Denisov V N, Marvin B N and Podobedov V B 1987 Phys. Rep. 1511

[52] Cyvin S J, Rauch J E and Decius J C 1965 J. Chem. Phys. 434083

[53] Tezuka Y, Shin S and Ishigami M 1991 Phys. Rev. Lett. 662356

[54] Taraskin S N and Elliott S R 1997 Phys. Rev. B 568605

[55] Guillot B and Guissani Y 1997 Phys. Rev. Lett. 782401

[56] Pasquarello A 2001 private communication

[57] Aslanyan T A and Levanyuk A P 1979 Solid State Commun. 31547

[58] Dolino G, Berge B, Vallade M and Moussa F 1992 J. Physique I 21461

[59] Akhieser A 1939 J. Phys. (USSR) 9277

[60] Bömmel H E and Dransfeld K 1960 Phys. Rev. B 1171245

[61] Wiedersich J, Adichtchev S V and Rössler E 2000 Phys. Rev. Lett. 842718

[62] Tagantsev S 2003 private communication

[63] Matic A, Engberg D, Masciovecchio C and Börjesson L 2001 Phys. Rev. Lett. 863803

[64] Matic A, Börjesson L, Ruocco G, Masciovecchio C, Mermet A, Sette F and Verbeni R 2001 Europhys. Lett. 54 77

[65] Taraskin S N and Elliott S R 2000 Phys. Rev. B 6112017

[66] Parshin D A 1994 Phys. Rev. B 499400

[67] Ramos M A and Buchenau U 1998 Tunnelling Systems in Amorphous and Crystalline Solids ed P Esquinazi (Berlin: Springer) ch 9 pp 527-69

[68] Parshin D A and Laermans C 2001 Phys. Rev. B 63132203 